Document downloaded from:

http://hdl.handle.net/10251/83433

This paper must be cited as:

Rueda, P.; Sánchez Pérez, EA. (2015). THE SUPPORT LOCALIZATION PROPERTY OF THE STRONGLY EMBEDDED SUBSPACES OF BANACH FUNCTION SPACES. Studia Scientiarum Mathematicarum Hungarica. 52(4):559-576. doi:10.1556/012.2015.1326.

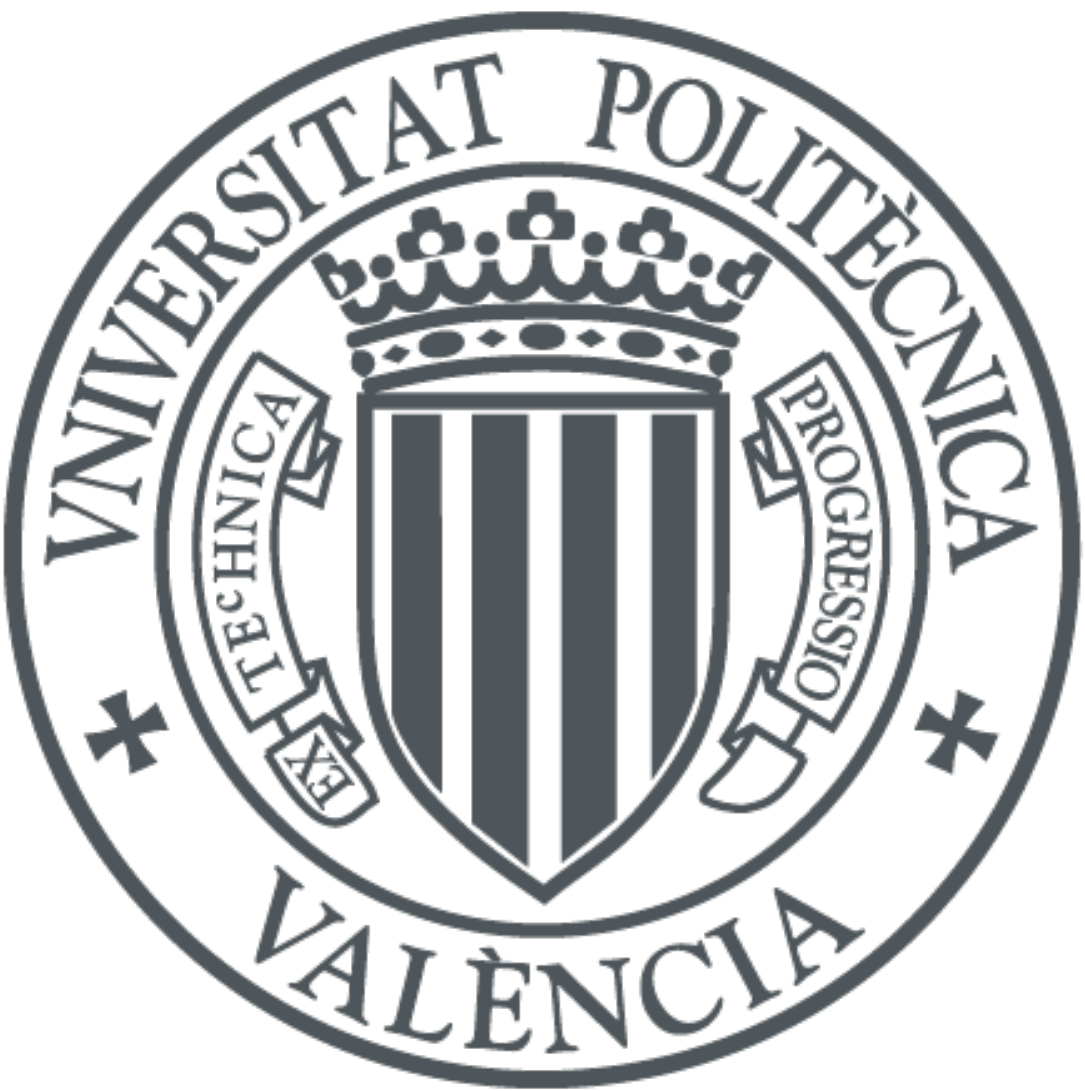

The final publication is available at

http://dx.doi.org/10.1556/012.2015.52.4.1326

Copyright AKADEMIAI KIADO RT

Additional Information

El archivo no es la versión final publicada del trabajo. 


\title{
THE SUPPORT LOCALIZATION PROPERTY OF THE STRONGLY EMBEDDED SUBSPACES OF BANACH FUNCTION SPACES
}

\author{
P. RUEDA AND E.A. SÁNCHEZ PÉREZ
}

\begin{abstract}
Motivated by the well known Kadec-Pełczynski disjointification theorem, we undertake an analysis of the supports of non-zero functions in strongly embedded subspaces of Banach functions spaces. The main aim is to isolate those properties that bring additional information on strongly embedded subspaces. This is the case of the support localization property, which is a necessary condition fulfilled by all strongly embedded subspaces. Several examples that involve Rademacher functions, the Volterra operator, Lorentz spaces or Orlicz spaces are provided.
\end{abstract}

\section{INTRODUCTION}

Let $(\Omega, \Sigma, \mu)$ be a finite measure space. Let $X(\mu)$ be an order continuous $p$-convex Banach function space $X(\mu)$ in the sense of [11, p.28]; in this case we have that $X(\mu) \subseteq L^{1}(\mu)$ continuously. A subspace $S$ of $X(\mu)$ is called strongly embedded - in $L^{1}(\mu)$ - if the restriction of the inclusion map $X(\mu) \rightarrow L^{1}(\mu)$ to $S$ is an isomorphism onto its range. The canonical example of such a subspace is the one generated by the Rademacher functions in $L^{p}[0,1]$, that is isomorphic to $\ell^{2}$ (for each $1 \leq p<\infty$ ) by Kintchine's inequalities (see Example 3(2)). The study of these and related subspaces of Banach function spaces has a long tradition (see for instance $[2,6,7,10]$ and the references therein). One of the main tools for analyzing these subspaces is the classical Kadec-Pełczynski disjointification method, that establishes that for subspaces of order continuous Banach function spaces, the following logical disjunction is given: either the subspace is strongly embedded in $L^{1}(\mu)$, or it contains a normalized sequence of (almost) disjoint functions (see [6, Th.4.1]). Of course, these statements are not mutually exclusive for every order continuous Banach function space over $\mu$ : a subspace of $L^{1}[0,1]$ being generated by a sequence of disjoint norm one functions satisfies both

2010 Mathematics Subject Classification. 46A50, 46B50, 46E30, 46G10.

Key words and phrases. Banach function space, vector measure integration, disjoint sequence, strongly embedded subspace.

P. Rueda acknowledges with thanks the support of the Ministerio de Economía y Competitividad (Spain) MTM2011-22417. E.A. Sánchez Pérez acknowledges with thanks the support of the Ministerio de Economía y Competitividad (Spain) MTM2012-36740-C0202 . 
statements. The analysis of such problem was initiated in the paper [9], in which subspaces of spaces $L^{p}[0,1], 1<p<\infty$, were studied. The requirement of $p$ being bigger than one is relevant for both cases to be exclusive; we will show in this paper that $p$-convexity for $p>1$ is the key requirement for getting such a result (see Proposition 1), and this will motivate a new property regarding the supports of the functions of a particular strongly embedded subspace. For the case of spaces not being $p$-convex for any $p>1$, it can be easily shown that the two cases of the Kadec-Pełczynski dichotomy can be satisfied simultaneously in certain subspaces. Besides the $L^{1}[0,1]$ case, already mentioned, we will show concrete examples (see the comments after Proposition 1).

Thus, the idea of this paper is to provide a new descriptive property for subspaces of order continuous Banach function spaces with non trivial $p$-convexity - the support localization property, SLP for short - that is satisfied by all the strongly embedded subspaces. We say that a subspace $S$ of a Banach function space has the support localization property if there is a $\delta>0$ such that for each finite family of functions in $S$ the characteristic functions of their supports $\chi_{A_{1}}, \ldots, \chi_{A_{n}}$ satisfy that $\delta n \leq\left\|\sum_{i=1}^{n} \chi_{A_{i}}\right\|_{X(\mu)}$.

We will center our attention in the case that $X(\mu)$ is a space $L^{p}(m)$ of $p$-integrable functions with respect to a vector measure $m$. This is not a real restriction of the general case of $p$-convex order continuous Banach lattices with a weak unit since these spaces are order and norm isomorphic to $L^{p}$ spaces of vector measures (see for example [5] or [13, Prop.3.30]). Therefore, the results concerning spaces of integrable functions with respect to a vector measure, as some in the present paper, can be applied to all order continuous Banach lattices with a weak unit and non trivial convexity. This is the reason for the recent effort that has been made by several authors in order to characterize strongly embedded subspaces of $L^{p}$ spaces of a vector measure (see [2]) and also the related class of the subspaces of a space that are fixed by the integration map (see [14]): the integration! structure of the $L^{p}$ spaces provides an additional tool for analyzing strongly embedded subspaces of general Banach lattices. However, we will also show that although the classes of strongly embedded subspaces and the one of the subspaces that are fixed by the integration map do not coincide on the spaces $L^{p}(m)$, both of them satisfy the support localization property under the assumption that $p>1$.

A related property that we will consider on a set of functions $S$-easier to understand from the point of view of the properties of the supports of the functions in $S$-, is the finite local support property, that is, the existence of a finite class $\mathcal{A}$ of measurable sets of positive measure such that the support of each (non-null) function in $S$ contains at least a set of $\mathcal{A}$.

The paper is organized as follows. After giving some preliminar material in Section 2, we introduce the support localization property and show that every strongly embedded subspace $S$ of $L^{p}(m)$ has it. The support localization property is related to the existence of an associated finite class $\mathcal{A}$ of sets of positive measure such that the support of each (non-null) function in 
$S$ contains at least a set of $\mathcal{A}$. In Section 4 we prove that every subspace of $L^{p}(m)$ fixed by the integration map is strongly embedded and thus satisfies the support localization property. Especial attention is paid to examples. These involve Rademacher functions, the Volterra operator, Lorentz spaces or Orlicz spaces.

\section{Preliminaries}

Our notation is standard; for $1 \leq p \leq \infty$ we write $p^{\prime}$ for the extended real number satisfying $1 / p+1 / p^{\prime}=1$. Throughout the paper, $E$ will be a Banach space, $B_{E}$ its unit ball and $S_{E}$ its unit sphere; $(\Omega, \Sigma)$ will be a measurable space and $m: \Sigma \rightarrow E$ a (countably additive) vector measure. For the definition of Banach function space we follow [11, Def.1.b.17, p.28], in the particular case of finite measure: given a finite measure space $(\Omega, \Sigma, \mu)$, an order ideal $X(\mu)$ of $L^{1}(\mu)$ is a Banach function space - called a Köthe function space in [11] - if it is a Banach space endowed with a norm $\|\cdot\|_{X(\mu)}$ that is compatible with the $\mu$-a.e. order. If the measure $\mu$ is not finite but $\sigma$ finite, the requirement $X(\mu) \subseteq L^{1}(\mu)$ is substituted by the local integrability of the functions in $X(\mu)$. The space $X(\mu)$ is order continuous if for each sequence in it decreasing $\mu$-a.e. to 0 , the corresponding sequence of the norms of the functions converges to 0 too. A Banach function space $X(\mu)$ is $p$-convex for $1 \leq p<\infty$ if there is a constant $K>0$ such that for each finite set of functions $f_{1}, \ldots, f_{n} \in X(\mu)$,

$$
\left\|\left(\sum_{i=1}^{n}\left|f_{i}\right|^{p}\right)^{1 / p}\right\|_{X(\mu)} \leq K\left(\sum_{i=1}^{n}\left\|f_{i}\right\|_{X(\mu)}^{p}\right)^{1 / p} .
$$

In the same way, $X(\mu)$ is said to be $p$-concave if the reverse inequality holds for some $K>0$. A normalized sequence $\left(f_{i}\right)_{i}$ of a Banach function space is said to be almost disjoint if there is a disjoint sequence $\left(z_{i}\right)_{i}$ such that $\lim _{i}\left\|f_{i}-z_{i}\right\|=0$.

Let us introduce now some notions regarding vector measures and integration. Let $E$ be a real Banach space and let $(\Omega, \Sigma)$ be a measurable space. Given a countably additive vector measure $m: \Sigma \rightarrow E$, we write $\mathcal{R}(m)$ for its range. Its variation $|m|$ is defined as

$$
|m|(A):=\sup _{\pi} \sum_{B \in \pi}\|m(B)\|,
$$

where the supremum is computed over all finite measurable partitions $\pi$ of $A \in \Sigma$. We write $\|m\|$ for its semivariation, that is defined by $\|m\|(A):=$ $\sup _{x^{*} \in B_{E^{*}}}\left|\left\langle m, x^{*}\right\rangle\right|(A), A \in \Sigma$, where $\left\langle m, x^{*}\right\rangle$ is the scalar measure given by $\left\langle m, x^{*}\right\rangle(A):=\left\langle m(A), x^{*}\right\rangle$. The reader can find in $[4,13]$ more information on vector measures and integration; see also [1] and the references therein for more information on the semivariation. It is known that there exists $x^{*} \in E^{*}$ such that $m$ is absolutely continuous with respect to $\left|\left\langle m, x^{*}\right\rangle\right|$, that is $m(A)=0$ whenever $\left|\left\langle m(A), x^{*}\right\rangle\right|=0$. Such a measure $\left\langle m, x^{*}\right\rangle$ is called a 
Rybakov measure for $m$; a fixed Rybakov measure $\mu$ for $m$ will be considered through the paper. For the aim of simplicity we will assume as usual that the element $x^{*}$ appearing in the definition of $\mu$ satisfies that $\left\|x^{*}\right\|=1$. If $1 \leq p<\infty$, a (scalar) measurable function $f$ is said to be $p$-integrable with respect to $m$ if two requirements hold: (1) $|f|^{p}$ is integrable with respect to all measures $\left|\left\langle m, x^{*}\right\rangle\right|$, and (2) for each $A \in \Sigma$ there exists an element $\int_{A}|f|^{p} d m \in E$ such that $\left\langle\int_{A}|f|^{p} d m, x^{*}\right\rangle=\int_{A}|f|^{p} d\left\langle m, x^{*}\right\rangle, x^{*} \in E^{*}$. For the case $p=\infty, L^{p}(m)$ is simple defined as $L^{\infty}(\mu)$ for a Rybakov measure $\mu$ for $m$ (obviously all Rybakov measures give the same space). The space $L^{p}(m), 1 \leq p<\infty$, is then given by the linear space of all $\mu$-equivalence classes of measurable real functions defined on $\Omega$ that are $p$-integrable with respect to $m$. It is a Banach function space over $\mu$ when the a.e. order and the norm

$$
\|f\|_{L^{p}(m)}:=\left(\sup _{x^{*} \in B_{E^{*}}} \int_{\Omega}|f|^{p} d\left|\left\langle m, x^{*}\right\rangle\right|\right)^{1 / p}, \quad f \in L^{p}(m),
$$

are considered. It is an order continuous $p$-convex Banach function space —with constant $K=1-($ see [16, Proposition 5]). Moreover, it is known that each order continuous $p$-convex Banach lattice with a weak unit is isomorphic in order and norm to an $L^{p}$ space of a vector measure (see [5] and $[13, \mathrm{Ch} .3]$ for this and for more information on these spaces).

A version of Hölder's inequality for these spaces is also available: if $f \in$ $L^{p}(m)$ and $g \in L^{p^{\prime}}(m),\|f g\|_{L^{1}(m)} \leq\|f\|_{L^{p}(m)} \cdot\|g\|_{L^{p^{\prime}(m)}}$. The integration operator $I_{m}: L^{1}(m) \rightarrow E$ is given by

$$
I_{m}(f)=\int_{\Omega} f d m, f \in L^{1}(m) .
$$

General information on the properties of $I_{m}$ can be found in [13] and the references therein. Since for all $p>1$ the inclusion $L^{p}(m) \subseteq L^{1}(m)$ always holds - as a consequence of Hölder's inequality - , the integration map can be considered also as an operator $I_{m}: L^{p}(m) \rightarrow E$.

\section{Strongly embedded subspaces of $L^{p}(m)$.}

Let $\mu$ be a finite (scalar) measure. As we said in the introduction, a subspace $S$ of a Banach function space $X(\mu)$ is strongly embedded if $S$ can be found isomorphically in $L^{1}(\mu)$. Since $S$ is always continuously included in $L^{1}(\mu)$, being strongly embedded is equivalent to saying that there is a $\delta>0$ such that $\int_{\Omega}|f| d \mu>\delta$ for all norm one $f \in S$. The most popular example of such a subspace is the linear span of the Rademacher functions, that we shall denote $\mathcal{R}$. Using Kintchine's inequalities, it is shown that $\mathcal{R}$ is strongly embedded in the spaces $L^{p}[0,1]$ for $1 \leq p<\infty$. It is well-known that this subspace is also strongly embedded in other spaces. For example, consider the closure $G$ of $L^{\infty}$ in the Orlicz space $L_{M_{2}}$ with $M_{2}(t):=e^{t^{2}}-1$. If $X(\mu)$ is a symmetric (or rearrangement invariant) Banach function space over Lebesgue measure $([0,1], \mathcal{B}, d x)$ and $G \subset X(\mu)$, then it is proved in [15] 
that $X(\mu)$ contains a copy of $\ell^{2}$ generated by $\mathcal{R}$. On the other hand, other examples of strongly embedded subspaces of Banach function spaces are available in the mathematical literature. For instance, given $1<p<s<2$, consider a sequence $\left(f_{i}\right)_{i}$ of independent and identically-distributed $s$-stable random variables in $L^{p}[0,1]$; the linear span is isometrically isomorphic to $\ell^{s}$ in this space and in $L^{1}[0,1]$. The reader can find more examples of strongly embedded subspaces of symmetric spaces in [12], where several examples of such subspaces are explained (see Theorem 4.3 in this paper).

Let us recall the Kadec-Pełczynski theorem regarding the behavior of the sequences in order continuous Banach function spaces (see [6, Th.4.1]). Consider the fixed Rybakov measure $\mu$ for $m$. Suppose that $S$ is a subspace of $L^{p}(m), p \geq 1$. Then we have that

(1) there is a $\delta>0$ such that $\int_{\Omega}|f| d \mu>\delta$ for all norm one $f \in S$, or

(2) there is a sequence $\left(f_{k}\right)_{k}$ of norm one elements of $S$ that is almost disjoint.

Thus, it may happen that either $S$ is a subspace also of $L^{1}(\mu)$ or it contains an almost disjoint normalized sequence. A priori, both situations can be given simultaneously, as it happens for instance for subspaces of $L^{1}(\mu)$ that are generated by a normalized sequence of disjointly supported functions in $L^{1}(\mu)$. Next result shows that these situations are mutually exclusive when $p>1$.

Proposition 1. Let $p>1$ and consider a subspace $S$ of $L^{p}(m)$. Then the following are equivalent.

(1) $S$ is strongly embedded in $L^{1}(\mu)$, where $\mu$ is a Rybakov measure for $m$.

(2) $S$ does not contain an almost disjoint sequence of norm one functions.

Proof. (1) $\Rightarrow(2)$. Assume that (2) does not hold, that is, there exists a sequence $\left(f_{i}\right)_{i}$ of norm one elements in $S$ which is almost disjoint. By (1), $S$ is isomorphic to a subspace of $L^{1}(\mu)$, the isomorphism given by the inclusion map. Then there is a constant $\delta>0$ such that

$$
\delta<\int_{\Omega}\left|f_{i}\right| d \mu \leq\left\|f_{i}\right\|_{L^{1}(m)} \leq\|m\|(\Omega)^{1 / p^{\prime}}\left\|f_{i}\right\|_{L^{p}(m)}=\|m\|(\Omega)^{1 / p^{\prime}},
$$

where Hölder's inequality for functions in the space $L^{1}(m)$ has been used. Choose a subsequence $\left(f_{i_{k}}\right)_{k}$ and a disjoint sequence $\left(g_{k}\right)_{k}$ in $L^{p}(m)$ such that for each $k \in \mathbb{N},\left\|f_{i_{k}}-g_{k}\right\|_{L^{p}(m)} \leq 1 / 2^{k}$. Note that

$$
\left|1-\left\|g_{k}\right\|_{L^{p}(m)}\right|=\left|\left\|f_{i_{k}}\right\|_{L^{p}(m)}-\left\|g_{k}\right\|_{L^{p}(m)}\right| \leq\left\|f_{i_{k}}-g_{k}\right\|_{L^{p}(m)} \leq 1 / 2^{k} .
$$

In particular, $\left\|g_{k}\right\|_{L^{p}(m)} \leq 2$ for each $k$.

Since the $g_{k}$ 's are pairwise disjoint then, $\sum_{k=1}^{\infty}\left|g_{k}\right|=\left(\sum_{k=1}^{\infty}\left|g_{k}\right|^{p}\right)^{1 / p}$. By the $p$-convexity of $L^{p}(m)$ and taking into account that $p>1$ we obtain for 
every $n$,

$$
\begin{gathered}
\delta n \leq \int_{\Omega} \sum_{k=1}^{n}\left|f_{i_{k}}\right| d \mu \leq\left\|\sum_{k=1}^{n}\left|f_{i_{k}}\right|\right\|_{L^{1}(m)} \leq\left\|\sum_{k=1}^{n}\left(\left|f_{i_{k}}-g_{k}\right|+\left|g_{k}\right|\right)\right\|_{L^{1}(m)} \\
\leq\|m\|(\Omega)^{1 / p^{\prime}} \sum_{k=1}^{n}\left\|f_{i_{k}}-g_{k}\right\|_{L^{p}(m)}+\left\|\left(\sum_{k=1}^{n}\left|g_{k}\right|^{p}\right)^{1 / p}\right\|_{L^{1}(m)} \\
\leq\|m\|(\Omega)^{1 / p^{\prime}} \sum_{k=1}^{n} \frac{1}{2^{k}}+\left\|\left(\sum_{k=1}^{n}\left|g_{k}\right|^{p}\right)^{1 / p}\right\|_{L^{p}(m)} \cdot\|m\|(\Omega)^{1 / p^{\prime}} \\
\leq\|m\|(\Omega)^{1 / p^{\prime}}+\left(\sum_{k=1}^{n}\left\|g_{k}\right\|_{L^{p}(m)}^{p}\right)^{1 / p} \cdot\|m\|(\Omega)^{1 / p^{\prime}} \leq\left(1+2 n^{1 / p}\right)\|m\|(\Omega)^{1 / p^{\prime}} .
\end{gathered}
$$

Consequently,

$$
\delta n^{1 / p^{\prime}} \leq\left(\frac{1}{n^{1 / p}}+2\right)\|m\|(\Omega)^{1 / p^{\prime}}
$$

which is impossible since the right hand tends to $2\|m\|(\Omega)^{1 / p^{\prime}}$ as $n$ tends to infinity. Thus, the sequence $\left(f_{i}\right)_{i}$ cannot be almost disjoint, and (2) holds.

For $(2) \Rightarrow(1)$, just apply the Kadec-Pełczynski result to the subspace $S$.

The requirement on the $p$-convexity of the space for $p>1$ is needed. Counterexamples to the proposition can be easily found by considering lattice copies of $\ell^{1}$ in $X(\mu)$. For instance, take a non atomic finite measure space $(\Omega, \Sigma, \mu)$ and consider a disjoint partition $\left(A_{i}\right)_{i}$ of non-null measurable subsets of $\Omega$. Let us write $\mu_{i}$ for the restricted measure to $A_{i}$ and take a family $\left\{X\left(\mu_{i}\right): i \in \mathbb{N}\right\}$ of Banach function spaces over $\mu_{i}$ such that the corresponding inclusions $X\left(\mu_{i}\right) \hookrightarrow L^{1}\left(\mu_{i}\right)$ have norm one and $\sum_{i=1}^{\infty}\left\|\chi_{A_{i}}\right\|_{X\left(\mu_{i}\right)}<\infty$. Define $X(\mu):=\bigoplus_{i, 1} X\left(\mu_{i}\right)$, that can be written as a Banach function space over $\mu$ with the obvious identification of the functions with their components in each $A_{i}$. It can be identified isometrically and in the order with a space $L^{1}(m)$, but not with an $L^{p}(m)$ space for any $p>1$, since it is not $p$-convex. equence $\left(\chi_{A_{i}}\right)_{i}$ gives a counterexample for this property, since $\chi_{\Omega}$ belongs to the space. Also, t The subspace of $X(\mu)$ generated by the sequence $\left(\chi_{A_{i}}\right)_{i}$ is strongly embedded in $L^{1}(\mu)$, and clearly it contains a disjoint sequence. An example of such a space is given by considering $X\left(\mu_{i}\right)=L^{p}\left(\mu_{i}\right)$, after renorming these spaces by $\|\cdot\|_{X\left(\mu_{i}\right)}=\mu\left(A_{i}\right)^{1 / p^{\prime}}\left\|f \chi_{A_{i}}\right\|_{L^{p}\left(\mu_{i}\right)}$. This example shows that there are more cases besides $L^{1}(\mu)$ spaces for which the two options in the Kadec-Pełczynski's dichotomy are not mutually exclusive. Other easy examples can be constructed by considering disjoint $p$-sums of $L^{1}(\mu)$ spaces, for $p>1$.

Let us show now some examples of spaces to which the previous result can be applied. 
Example 2. Let $1 \leq r<\infty$ and let $L^{r}[0,1]$ be the Lebesgue space of $r$ integrable functions. Let us write $([0,1], \mathcal{B}([0,1]), d x)$ for the corresponding measure space. Consider the Volterra operator $V_{r}: L^{r}[0,1] \rightarrow L^{r}[0,1]$, that is given by $V_{r}(f)(t):=\int_{[0, t]} f(x) d x$. It defines a countably additive vector measure $\nu_{r}$ by $\nu_{r}(A):=V_{r}\left(\chi_{A}\right), A \in \Sigma$ (see Example 3.26 and Example 3.49 in [13]). In the case of $r=1$, the space $L^{1}\left(\nu_{1}\right)$ coincides with $L^{1}\left(\left|\nu_{1}\right|\right)$ and so it is an $L^{1}$ space of a scalar measure, although the integration map is far from being of finite rank (in fact, it is not even weakly compact, see Example 3.49(iv) in [13]). Then (1) and (2) of Proposition 1 cannot be exclusive for $L^{1}\left(\nu_{1}\right)$, but it is so if $S$ is a subspace of $L^{p}\left(\nu_{1}\right), 1<p<\infty$, since it coincides (topologically and in the order) with $L^{p}\left(\left|\nu_{1}\right|\right)$. However, for $r>1$ the space $L^{1}\left(\nu_{r}\right)$ does not coincide with $L^{1}\left(\left|\nu_{r}\right|\right)$, and so gives a different Banach function space over any Rybakov measure for $\nu_{r}$ (see Example 3.26 in [13] for the description of this space). Proposition 1 can then be applied to subspaces of $L^{p}\left(\nu_{r}\right)$ for every $1<p<\infty$.

Example 3. Consider the Lebesgue measure space $([0,1], \mathcal{B}([0,1]), d x)$. Take $1<p<\infty$ and consider two vector measures defined as follows.

(1) The first case is defined simply as $m: \Sigma \rightarrow L^{1}[0,1]$ given by $m(A):=$ $\chi_{A}, A \in \mathcal{B}([0,1])$. In this case, it is well-known that the integration operator is the identity map, $L^{1}(m)=L^{1}[0,1]$ and so $L^{p}(m)=L^{p}[0,1]$. Moreover, Lebesgue measure can be considered in fact as a Rybakov measure $\mu$ for $m$, and $L^{1}(\mu)$ coincides also with $L^{1}[0,1]$ (see Example 3.61 in [13]).

(2) The second vector measure that we consider takes its values in $c_{0}$ and is given by the formula $m_{0}(A):=\left(\int_{A} r_{k-1}(t) d t\right)_{k=1}^{\infty}$, where $r_{0}=\chi_{[0,1]}, r_{j}$ is the $j$-th Rademacher function, $j \geq 1$, and $A \in \mathcal{B}([0,1])$. A direct computation shows that $L^{1}\left(m_{0}\right)$ and $L^{1}[0,1]$ coincide; in particular, Lebesgue measure is also equivalent to $m_{0}$.

In both cases, let $\mathcal{R}_{0}$ be the Rademacher functions and $\mathcal{R}$ the subspace generated by $\mathcal{R}_{0}$ in $L^{1}[0,1]$; due to Kintchine's inequalities, this space can also be found (isomorphically) in $L^{p}[0,1]$. Therefore, Proposition 1 implies that $\mathcal{R}$ does not contain an almost disjoint sequence of functions.

Consider a Banach function space $X(\mu)$ over a finite measure $\mu$. Example 3 concerning Rademacher functions is our motivation to analyze what happens with the supports of the functions of the strongly embedded subspaces of a Banach function space $X(\mu)$. We will see that being $\mathcal{R}$ strongly embedded is deeply related to the fact that the support of each Rademacher function is $[0,1]$ and so, to the fact that the support of Rademacher functions intersect on a set of positive measure.

Consider a Banach function space $X(\mu)$ over a finite measure $\mu$.

Definition 4. We say that a subset $S$ of $X(\mu)$ has finite local support if there is a finite disjoint class of sets $A_{1}, \ldots, A_{n}$ with positive measure such that for each $0 \neq f \in S$ there is $i_{0} \in\{1, \ldots, n\}$ such that $A_{i_{0}} \subseteq \operatorname{supp}\{f\}$. 
Trivially, $\mathcal{R}_{0}$ has finite local support. A more sofisticated property related to the supports of the functions in a set $S$ that will give us information on the strongly embedded subspaces is the support localization property:

Definition 5. Let $S$ be a subspace of $X(\mu)$. If there is a constant $0<\delta$ such that for every finite set of non zero functions $f_{1}, \ldots, f_{n} \in S$, the characteristic functions of their supports $\chi_{\operatorname{supp}\left\{f_{1}\right\}}, \ldots, \chi_{\operatorname{supp}\left\{f_{n}\right\}}$ satisfy that

$$
\delta n \leq\left\|\sum_{i=1}^{n} \chi_{\text {supp }\left\{f_{i}\right\}}\right\|_{X(\mu)},
$$

we will say that $S$ has the support localization property (SLP for short).

Remark 6. The following immediate property provides some additional information and will be used several times: A subspace having the support localization property cannot contain a disjoint sequence. To see this, suppose that there is a disjoint normalized sequence $\left(f_{i}\right)_{i}$ in an arbitrary subspace $S$ of $L^{p}(m)$. If $A_{i}$ is the support of $f_{i}$ for each $i$, then $\left\|\sum_{i=1}^{n} \chi_{A_{i}}\right\|_{L^{p}(m)} \leq$ $\left\|\chi_{\Omega}\right\|_{L^{p}(m)} \leq\|m\|(\Omega)^{1 / p}$, and then the left hand side of these inequalities cannot increase with $n$.

Note that the inequality (1) applied to each function $f \in S$ gives then $\delta \leq\left\|\chi_{\operatorname{supp}\{f\}}\right\|_{L^{p}(m)}$. An easy calculation shows that $\|m\|(\operatorname{supp}\{f\}) \geq \delta^{p}$ for all $f \in S$, that is, the SLP implies that the measures of the supports of all non-zero functions in $S$ must be uniformly bounded below.

Let us show in the next proposition that having finite local support implies the SLP. A sort of converse under an additional requirement is also true. Remark 6 will be used in the following proof.

Proposition 7. Let $S$ be a subspace of $X(\mu)$.

(i) If $S \backslash\{0\}$ has finite local support, then $S$ has the $S L P$.

(ii) Let $S_{0} \subseteq S \backslash\{0\}$ be such that for each $f \in S \backslash\{0\}$ and $g \in S_{0}$, $f \chi_{\text {supp }\{g\}} \in S$ and assume that $S$ has the $S L P$. Then $S_{0}$ has finite local support.

Proof. (i) Let $A_{1}, \ldots, A_{n}$ be given according to the finite local support property for $S \backslash\{0\}$. Consider a finite family of nonzero functions $f_{1}, \ldots, f_{m} \in S$. For each $i \in\{1, \ldots, m\}$, there exists $j_{i} \in\{1, \ldots, n\}$ such that $A_{j_{i}} \subset$ $\operatorname{supp}\left\{f_{i}\right\}$. Let $K>0$ be such that $K\|g\|_{L^{1}(\mu)} \leq\|g\|_{X(\mu)}$ for all $g \in X(\mu)$. Then,

$$
\begin{aligned}
m K \min _{1 \leq j \leq n}\left\|\chi_{A_{j}}\right\|_{L^{1}(\mu)} & \leq K \sum_{i=1}^{m}\left\|\chi_{A_{j_{i}}}\right\|_{L^{1}(\mu)}=K\left\|\sum_{i=1}^{m} \chi_{A_{j_{i}}}\right\|_{L^{1}(\mu)} \\
& \leq\left\|\sum_{i=1}^{m} \chi_{A_{j_{i}}}\right\|_{X(\mu)} \leq\left\|\sum_{i=1}^{m} \chi_{\operatorname{supp}\left\{f_{i}\right\}}\right\|_{X(\mu)} .
\end{aligned}
$$

Consequently, the SLP holds for $\delta:=K \min _{i=1, \ldots, n}\left\|\chi_{A_{i}}\right\|$. 
(ii) Assume that $S_{0}$ has not finite local support. Consider the class of all families of disjoint non-zero functions of $S$ ordered by inclusion (the "first" order considered in the arguments below). By Remark 6 each family is necessarily finite; otherwise we would have a contradiction with the SLP of $S$. Besides, a similar argument shows that each chain of families is also finite. Zorn's Lemma guaranties that the set $\mathcal{M}$ of maximal families in the class is non-empty.

Define now a different order (the "second" order) in the set $\mathcal{M}$, given by the following relation: a (finite) set $\mathcal{F}_{1}:=\left\{h_{1}, \ldots, h_{m}\right\} \in \mathcal{M}$ is less than a (finite) set $\mathcal{F}_{2}=\left\{g_{1}, \ldots, g_{k}\right\} \in \mathcal{M}$ if $A=\cup_{i=1}^{m} \operatorname{supp}\left\{h_{i}\right\}=\cup_{i=1}^{k} \operatorname{supp}\left\{g_{i}\right\}$ and the partition of $A$ defined by the supports of the elements of $\mathcal{F}_{2}$ is finer than the one defined by $\mathcal{F}_{1}$. Again, the chains contain only a finite number of elements, since otherwise we get a contradiction with the SLP. Applying Zorn's Lemma again we obtain a maximal element $\mathcal{F}=\left\{f_{1}, \ldots, f_{n}\right\}$ with respect to the new order, and also (by the construction) with respect to the order given in the previous paragraph.

Now recall that we are assuming that $S_{0}$ has not finite local support. Therefore, there is a function $g \in S_{0}$ such that $\operatorname{supp}\left\{f_{i}\right\}$ is not included in $\operatorname{supp}\{g\}$ for any $i$. We have now two possible cases.

a) $\operatorname{supp}\left\{f_{i}\right\} \cap \operatorname{supp}\{g\}=\emptyset$ for all $i=1, \ldots, n$. Then the maximality of $\mathcal{F}$ with respect to the first order gives a contradiction with the existence of the family of disjoint functions $\mathcal{F} \cup\{g\}$.

b) There is an index $i_{0}$ such that $\operatorname{supp}\left\{f_{i_{0}}\right\} \cap \operatorname{supp}\{g\} \neq \emptyset$. Then by hypothesis we have that $h_{1}:=f_{i_{0}} \chi_{\text {supp }\{g\}} \in S$, and so $h_{2}:=f_{i_{0}}-f_{i_{0}} \chi_{\text {supp }\{g\}} \in$ $S$ too. If we consider the family defined by $\left(\mathcal{F} \backslash\left\{f_{i_{0}}\right\}\right) \cup\left\{h_{1}, h_{2}\right\}$, we get a contradiction with the maximality of $\mathcal{F}$ with respect to the second order relation. This finishes the proof.

Part (ii) of this result can be used to prove that having finite local support and having the support localization property are equivalent properties for some types of subspaces. For instance, take $B$ the set of all functions with support equal to $\Omega$. Take also a family of measurable subsets $A=\left\{A_{i}\right.$ : $i \in I\}$ such that for each $i, j \in I, A_{i} \cap A_{j} \in A$ and consider the subspace $S$ generated by the pointwise product $B \cdot\left\{\chi_{A_{i}}: i \in I\right\}$. Then a straightforward argument using the result shows that $S_{0}:=S \backslash\{0\}$ has finite local support if and only if $S$ has the support localization property.

Following with our main example, given by $\mathcal{R}$, we have that $\mathcal{R}_{0}$ has finite local support. However, the above result does not provide a proof of the fact that $\mathcal{R}$ has the SLP. We will prove in the next result that every strongly embedded subspace of $L^{p}(m)$ has the support localization property. In particular, $\mathcal{R}$ has the SLP.

Theorem 8. Let $p>1$ and consider a subspace $S$ of $L^{p}(m)$. If $S$ is strongly embedded then $S$ has the support localization property. 
Proof. If $S$ is strongly embedded in $L^{1}(\mu)$, where $\mu$ is a Rybakov measure for $m$, then there exists $\delta>0$ such that

$$
\delta\|f\|_{L^{p}(m)}<\int_{\Omega}|f| d \mu \leq\|f\|_{L^{1}(m)} \leq\|f\|_{L^{p}(m)}\|m\|(\Omega)^{1 / p^{\prime}}
$$

for every $f \in S$. Consider $\left(g_{i}\right)_{i}$ a sequence in $S, g_{i} \neq 0$ for all $i$ and let $\left(A_{i}\right)_{i=1}^{n}$ be the sequence of supports of the functions $g_{i}$. Consider the related sequence of normalized functions $f_{i}=g_{i} /\left\|g_{i}\right\|$. Obviously, the support of each such function $f_{i}$ is $A_{i}$. By the $p$-convexity of $L^{p}(m)$ and taking into account that $p>1$ we obtain for every $n$,

$$
\begin{aligned}
\delta n & \leq \int_{\Omega} \sum_{i=1}^{n}\left|f_{i}\right| d \mu \leq\left\|\sum_{i=1}^{n}\left|f_{i}\right|\right\|_{L^{1}(m)}=\left\|\sum_{i=1}^{n}\left|f_{i}\right| \chi_{A_{i}}\right\|_{L^{1}(m)} \\
& \leq\left\|\left(\sum_{i=1}^{n}\left|f_{i}\right|^{p}\right)^{1 / p}\left(\sum_{i=1}^{n} \chi_{A_{i}}\right)^{1 / p^{\prime}}\right\|_{L^{1}(m)} \\
& \leq\left\|\left(\sum_{i=1}^{n}\left|f_{i}\right|^{p}\right)^{1 / p}\right\|_{L^{p}(m)}\left\|\left(\sum_{i=1}^{n} \chi_{A_{i}}\right)^{1 / p^{\prime}}\right\|_{L^{p^{\prime}(m)}} \\
& \leq\left(\sum_{i=1}^{n}\left\|f_{i}\right\|_{L^{p}(m)}^{p}\right)^{1 / p}\left\|\sum_{i=1}^{n} \chi_{A_{i}}\right\|_{L^{1}(m)}^{1 / p^{\prime}} \\
& \leq n^{1 / p}\left\|\sum_{i=1}^{n} \chi_{A_{i}}\right\|_{L^{1}(m)}^{1 / p^{\prime}}
\end{aligned}
$$

Consequently, $\delta^{p^{\prime}} n \leq\left\|\sum_{i=1}^{n} \chi_{A_{i}}\right\|_{L^{1}(m)} \leq\left\|\sum_{i=1}^{n} \chi_{A_{i}}\right\|_{L^{p}(m)}\|m\|(\Omega)^{1 / p^{\prime}}$.

Note that the requirement $p>1$ is again needed in the previous result, although the Kadec-Pełczynski's dichotomy works for general order continuous Banach function spaces. For example, take a disjoint sequence $\left(A_{i}\right)_{i}$ of measurable sets in $[0,1]$ of non-zero measure, and the subspace $S$ generated by the characteristic functions of these sets in $L^{1}[0,1]$. Obviously, this space is strongly embedded in $L^{1}[0,1]$, but there is no $\delta>0$ such that

$$
\delta n \leq\left\|\sum_{i=1}^{n} \chi_{A_{i}}\right\|_{L^{1}[0,1]} \leq 1 .
$$

Example 9. Examples and characterizations of strongly embedded subspaces of Lorentz spaces were studied in the classical papers [3] and [6]. More information on the strongly embedded subspaces of Lorentz $L^{p, q}$ spaces can be found in [17] (see also [8]). Consider the generalized Lorentz spaces $\Lambda(W, p)$ studied in Section 5 of [6]. This is the order continuous Banach function space (over the Lebesgue measure) of all the (classes of) measurable functions $f$ on $[0,1]$ for which $\left(\int_{[0,1]} f^{*}(t)^{p} W(t) d t\right)^{1 / p}<\infty$, where $f^{*}$ is the 
decreasing rearrangement of $f$ and $W$ is a non-bounded non-increasing function on $(0,1]$ which integral equals 1 and $W(1)>0$. Then $\Lambda(W, p) \subseteq L^{p}[0,1]$, and the results in [6] show that a subspace of $\Lambda(W, p)$ either embeds isomorphically into $L^{p}[0,1]$ or contains a complemented subspace isomorphic to $\ell_{p}$ (see Remark 5.6 in [6]). Therefore, strongly embedded subspaces of these Lorentz spaces are closely related to the ones of $L^{p}[0,1]$. In particular, for the case of a subspace $S$ of the Lorentz spaces $L^{p, q}[0,1], 1<p<\infty$ and $1 \leq q<\infty$-in fact, for all the subspaces of $L^{p, q}[0, \infty)$ - it happens that it can be either isomorphic to a strongly embedded subspace of $L^{p}[0,1]$ or contains a complemented copy of $\ell_{q}$ (Corollary 2.9 in [3]). Therefore, Proposition 1 may be applied to show that the strongly embedded subspaces of the Lorentz spaces $L^{p, q}[0, \infty)$ with $1<p<\infty$ and $1 \leq q<\infty$ not containing copies of $\ell_{q}$ have the SLP. A similar situation will be shown in the last example of the present paper, where copies of $\ell_{q}$ become crucial.

The result for general Banach function spaces can be written as follows.

Corollary 10. If $X(\mu)$ is a Banach function space with

i. non trivial convexity and concavity, or

ii. $p$-convexity for $p>1$ and finite cotype, or

iii. type strictly bigger than 1 ,

then each strongly embedded subspace of $X(\mu)$ has the support localization property.

Proof. First recall that having finite concavity (or finite cotype) implies that $X(\mu)$ is order continuous; this is a consequence of a direct calculation. For the equivalence between nontrivial concavity and finite cotype in a Banach lattice see [11, p.74], [11, Prop. 1.f.3], [11, Cor.1.f.9] and in general [11, Sec.1.f]. Moreover, if the type of $X(\mu)$ is $>1$, then by [11, Cor.1.f.13] it is $q$-concave for some $q<\infty$ and by [11, Cor.1.f.9], it is also $p$-convex for some $p>1$. The result is then a consequence of Theorem 8 and the fact that each order continuous $p$-convex space is order isomorphic to an $L^{p_{-}}$ space of a vector measure $m$, for which $\mu$ is a Rybakov measure (see [13, Prop.3.30]).

Let $X(\mu)$ be a Banach function space and let $Y$ be a Banach space. An operator $T: X(\mu) \rightarrow Y$ is disjointly strictly singular if the restriction of $T$ to any subspace generated by a sequence of non-zero disjoint functions is not an isomorphism. In [12, Theorem 3.2] the following result is proved.

Theorem 11. [12, Theorem 3.2] If $X(\mu)$ is a symmetric Banach space such that $X(\mu) \neq L^{1}(\mu)$, then the inclusion $i: X(\mu) \rightarrow L^{1}(\mu)$ is disjointly strictly singular.

The next corollary shows that a similar result can be obtained for order continuous $p$-convex Banach function spaces with weak unit. Note that there is a subspace $Y$ generated by a sequence of non-zero disjoint functions in 
$X(\mu)$ that is strongly embedded in $L^{1}(\mu)$ if and only if the inclusion map $i: X(\mu) \rightarrow L^{1}(\mu)$ is not disjointly strictly singular.

Corollary 12. Let $1<p<\infty$. Let $m$ be a vector measure and $\mu$ a Rybakov measure for $m$. The inclusion map $i: L^{p}(m) \rightarrow L^{1}(\mu)$ is disjointly strictly singular.

Proof. If $i$ was not disjointly strictly singular, then there is a subspace $Y$ generated by a sequence of non-zero disjoint functions in $L^{p}(m)$ such that $i: Y \rightarrow L^{1}(\mu)$ is an isomorphism. Then by Remark $6 Y$ does not have the SLP, and so by Theorem $8, Y$ is not strongly embedded, a contradiction.

\section{Applications: subspaces that are fixed By the integration MAP}

Recently, some effort has been made in order to analyze the subspaces of the spaces $L^{1}(m)$ that are fixed by the integration map, i.e. those for which the restriction of this operator is an isomorphism (see [14] and the references therein). In this section we consider the same property but for subspaces of $L^{p}(m), 1<p<\infty$, in order to prove that all the spaces that are fixed isomorphically by the integration map are strongly embedded. In particular, these have the SLP. We also provide some examples for proving that both classes of subspaces satisfying these properties do not coincide.

Lemma 13. If $m: \Sigma \rightarrow E$ is a Banach space valued (countably additive) vector measure, then

$$
\lim _{n} \frac{\sum_{i=1}^{n}\|m\|\left(A_{i}\right)}{n}=0
$$

for every disjoint sequence of measurable sets $\left(A_{i}\right)_{i}$ in $\Sigma$.

Proof. Consider such a sequence of measurable sets and fix an $\varepsilon>0$. Then, using the equivalent expression for the semivariation of a vector measure given in Proposition 11 of [4, Ch.I] we have that for each $i$ there is a measurable set $B_{i} \subset A_{i}$ such that $\|m\|\left(A_{i}\right) \leq 2\left\|m\left(B_{i}\right)\right\|+\varepsilon / 2^{i}$. On the other hand, take a Rybakov measure $\mu$ for $m,[4$, Ch.IX]. Since $\mu$ is finite and the elements in $\left(B_{i}\right)_{i}$ are disjoint, we obtain that $\lim _{i} \mu\left(B_{i}\right)=0$. Thus, $\lim _{i} m\left(B_{i}\right)=0$ (see for example Pettis Theorem [4, Th.1,Ch.I]). Now it is enough to apply Stolz criterium for computing the $\operatorname{limit}_{\lim _{n}} a_{n} / n$, with $a_{n}=\sum_{i=1}^{n}\left(2\left\|m\left(B_{i}\right)\right\|+\varepsilon / 2^{i}\right)$;

$$
\lim _{n} \frac{a_{n+1}-a_{n}}{n+1-n}=\lim _{n} 2\left\|m\left(B_{n+1}\right)\right\|+\varepsilon / 2^{n+1}=0,
$$

and so $\lim _{n} a_{n} / n=0$. Consequently,

$$
\lim _{n} \frac{\sum_{i=1}^{n}\|m\|\left(A_{i}\right)}{n} \leq \lim _{n} \frac{\sum_{i=1}^{n}\left(2\left\|m\left(B_{i}\right)\right\|+\varepsilon / 2^{i}\right)}{n}=0
$$

what proves the lemma. 
Theorem 14. Let $p>1$ and let $S$ be a subspace of $L^{p}(m)$. If $S$ is fixed isomorphically by the integration map $I_{m}: L^{p}(m) \rightarrow E$, then $S$ is strongly embedded in $L^{1}(\mu)$.

Proof. Consisder a subspace $S$ as in the statment of the theorem. Since it is fixed isomorphically by the integration map $I_{m}: L^{p}(m) \rightarrow E$, there is a constant $\delta>0$ such that

$$
\delta\|f\|_{L^{p}(m)} \leq\left\|\int_{\Omega} f d m\right\|_{E} \leq\|f\|_{L^{1}(m)} \leq\|m\|(\Omega)^{1 / p^{\prime}}\|f\|_{L^{p}(m)}
$$

for all $f \in S$.

Assume that there is a sequence $\left(f_{i}\right)_{i}$ in $S$ with $\left\|f_{i}\right\|=1$ for all $i$, and a disjoint sequence $\left(g_{i}\right)_{i}$ in $L^{p}(m)$ such that $\lim _{i}\left\|f_{i}-g_{i}\right\|_{L^{p}(m)}=0$. For each $i$, let $A_{i}$ be the support of $g_{i}$. For $\varepsilon>0$ we find a subsequence $\left(g_{i_{k}}\right)_{k}$ such that $\left\|f_{i_{k}}-g_{i_{k}}\right\|_{L^{p}(m)} \leq \varepsilon / 2^{k}$ for all $k$. As in the proof of Proposition 1, we can assure that $\left\|g_{i_{k}}\right\| \leq 2$ for every $k$. Then, for each $n$,

$$
\begin{aligned}
\delta n & \leq \sum_{k=1}^{n}\left\|\int_{\Omega} f_{i_{k}} d m\right\|_{E} \leq \sum_{k=1}^{n}\left\|g_{i_{k}}\right\|_{L^{1}(m)}+\sum_{k=1}^{n}\left\|f_{i_{k}}-g_{i_{k}}\right\|_{L^{1}(m)} \\
& \leq \sum_{k=1}^{n}\left\|g_{i_{k}} \chi_{A_{i_{k}}}\right\|_{L^{1}(m)}+\varepsilon \sum_{k=1}^{n} \frac{1}{2^{k}}\|m\|(\Omega)^{1 / p^{\prime}} \\
& \leq\left(\sum_{k=1}^{n}\left\|g_{i_{k}}\right\|_{L^{p}(m)}^{p}\right)^{1 / p} \cdot\left(\sum_{k=1}^{n}\|m\|\left(A_{i_{k}}\right)\right)^{1 / p^{\prime}}+\|m\|(\Omega)^{1 / p^{\prime}} \varepsilon \\
& \leq 2 n^{1 / p}\left(\sum_{k=1}^{n}\|m\|\left(A_{i_{k}}\right)\right)^{1 / p^{\prime}}+\|m\|(\Omega)^{1 / p^{\prime}} \varepsilon .
\end{aligned}
$$

Hence,

$$
\delta n^{1 / p^{\prime}} \leq 2\left(\sum_{k=1}^{n}\|m\|\left(A_{i_{k}}\right)\right)^{1 / p^{\prime}}+\frac{\|m\|(\Omega)^{1 / p^{\prime}} \varepsilon}{n^{1 / p}}
$$

and so

$$
\delta^{p^{\prime}} \leq \frac{1}{n}\left(2\left(\sum_{k=1}^{n}\|m\|\left(A_{i_{k}}\right)\right)^{1 / p^{\prime}}+\frac{\|m\|(\Omega)^{1 / p^{\prime}} \varepsilon}{n^{1 / p}}\right)^{p^{\prime}} .
$$

This contradicts Lemma 13. Therefore, such a sequence $\left(f_{i}\right)_{i}$ cannot exist and Proposition 1 gives the result.

Example 15. Consider the situation described in Example 3 for $r=1$ i.e. the vector measure $m: \Sigma \rightarrow L^{1}[0,1]$ given by $m(A):=\chi_{A}$. We obtain the following direct consequence of Theorem 14: for $p>1$, each subspace $S$ of $L^{p}(m)=L^{p}[0,1]$ that is fixed by the integration map - that in this case is simply the inclusion of $L^{p}[0,1]$ in $L^{1}[0,1]$, see Example 3.61 and Corollary 3.66(ii) in [13] - , satisfies the support localization property. For instance, this is the case of $S=\mathcal{R}$, the space generated by the Rademacher functions, that is fixed by the integration map when restricted to $L^{p}(m)$. 
In fact, is not difficult to find subspaces of spaces $L^{p}[0,1]$ others than $\mathcal{R}$ that are fixed by the integration map, and so has the SLP. For instance, Lemma 1.4 in [7] can be used. This result states the following: Let $\left(f_{n}\right)_{n}$ be a weakly null normalized sequence in $L^{p}(\mu)$ for some finite measure $\mu$ and $1<p<\infty$. Suppose that $\left(f_{n}\right)_{n}$ is uniformly bounded (that is, there exists $M<\infty$ such that $\left|f_{n}\right| \leq M$. Then, there is a subsequence $\left(f_{n_{k}}\right)_{k}$ equivalent to the unit vector basis of the sequence space $\ell_{2}$. Let $(\Omega, \Sigma, \mu)$ be a finite measure space. Let us show how to get a subspace that can be found simultaneously in $L^{p}(\mu)$ and $L^{q}(\mu), 1<p \leq q<\infty$. Note that $L^{q}(\mu) \subseteq$ $L^{p}(\mu)$. Take a uniformly bounded normalized weakly null sequence $\left(f_{n}\right)_{n}$ in $L^{q}(\mu)$. Then there is a subsequence $\left(f_{n_{k}}\right)_{k}$ satisfying that is equivalent to the unit vector basis of $\ell_{2}$, and the subspace $G$ generated by $\left(f_{i_{k_{j}}}\right)_{j}$ is weakly null also in $L^{p}(\mu)$. Thus, there is a subsequence $\left(f_{i_{k_{j}}}\right)_{j}$ that is also equivalent to the unit vector basis of $\ell_{2}$, and so it can be found simultaneously in $L^{p}(\mu)$ and $L^{q}(\mu)$. Consider the vector measure $m_{p}: \Sigma \rightarrow L^{p}(\mu)$ defined as above by $m_{p}(A):=\chi_{A}$. Then $L^{1}\left(m_{p}\right)=L^{p}(\mu)$ and $L^{r}\left(m_{p}\right)=L^{q}(\mu)$, for $r=q / p \geq 1$, and each subspace $G$ can be found in both $L^{1}\left(m_{p}\right)$ and $L^{r}\left(m_{p}\right)$, being the inclusion map $i: L^{q}(\mu) \rightarrow L^{p}(\mu)$ the operator giving the isomorphism. Of course, the subspace is fixed by the integration map $I_{m_{p}}: L^{1}\left(m_{p}\right) \rightarrow L^{p}(\mu)$, that is just the identity map.

Let us finish the paper with some counterexamples regarding the relation between strongly embedded subspaces and subspaces that are fixed by the integration map. Our aim is to illustrate that for $p>1$ the class of all the subspaces that are fixed isomorphically by the integration map does not coincide with the class of all the subspaces that can be found both in $L^{1}(m)$ and $L^{p}(m)$.

First, notice the following fact: Let $S$ be a subspace of $L^{p}(m)$ such that $\left.I_{m}\right|_{S}$ is an isomorphism into. Then $\left(S,\|\cdot\|_{L^{p}(m)}\right)$ is also isomorphic to $\left(S,\|\cdot\|_{L^{1}(m)}\right)$. This is just a consequence of the factorization of $I_{m}: L^{p}(m) \rightarrow$ $E$ through the inclusion $L^{p}(m) \hookrightarrow L^{1}(m)$.

Example 16. Fix $1 \leq q<\infty$. Consider Lebesgue measure space $([0,1], \mathcal{B}, d x)$ and an absolutely summable sequence of (non null) positive integrable functions $\left(f_{i}\right)_{i} \subseteq L^{1}[0,1]$ that satisfies that the union of the supports of all of them covers $[0,1]$. Define now the vector measure $\nu: \mathcal{B}([0,1]) \rightarrow \ell^{q}$ by $\nu(A):=\sum_{i=1}^{\infty} \int_{A} f_{i} d x \cdot e_{i} \in \ell^{q}$, where $\left\{e_{i}\right\}_{i=1}^{\infty}$ is the canonical basis. For $1 \leq p<\infty$ the norm for each function $f \in L^{p}(\nu)$ is given by

$$
\|f\|_{L^{p}(\nu)}:=\left(\sum_{i=1}^{\infty}\left(\int_{[0,1]}|f|^{p} f_{i} d x\right)^{q}\right)^{1 / q p} .
$$

(1) There are subspaces that are found simultaneously in $L^{p}(\nu), L^{1}(\nu)$ and $L^{1}[0,1]$ but are not fixed by the integration map. Suppose that $\left(f_{i}\right)_{i}$ satisfies that $\left\|\chi_{[0,1]}-2^{i} f_{i}\right\|_{L^{\infty}(\mu)}<1 / 2$ for all $i \in \mathbb{N}$. Then it can be checked that the norm of $L^{1}(\nu)$ is equivalent to the one in $L^{1}[0,1]$ but the integration map is 
not an isomorphism; this can be easily seen if for example each $f_{i}$ is defined as $1 / 2^{i} \chi_{[0,1]}$. Indeed, the range of the integration map has dimension 1 , and so it cannot be an isomorphism when restricted to any infinite dimensional subspace. However, $L^{p}(\nu)=L^{p}[0,1]$ and the subspaces that can be found simultaneously in $L^{p}(\nu)$ and $L^{1}(\nu)$-for example, the one spanned by the Rademacher functions-, are also in $L^{1}(\xi)$ for each Rybakov measure $\xi$, since $L^{1}(\xi)=L^{1}[0,1]$ isomorphically.

(2) There are subspaces that are fixed by the integration map $I_{\nu}: L^{1}(\nu) \rightarrow$ $E$ and that are simultaneously subspaces of $L^{1}(\nu)$ and $L^{1}[0,1]$, but that are not subspaces of $L^{p}(\nu)$ for $p>1$. Assume now that the functions $\left(f_{i}\right)_{i}$ are disjoint and fix a finite subset $N \subset \mathbb{N}$. Then there is no infinite dimensional subspace of functions of $L^{p}(\nu)$ with support in $\cup_{i \in N} A_{i}$ for any $1 \leq p<\infty$ that is fixed isomorphically by the integration map, since all the integrals of such functions lie in a finite dimensional subspace of $\ell^{q}$. However, $L^{1}(\nu)$ contains a copy $S$ of $\ell^{q}(\eta)$ that is fixed by the integration map, where the weight $\eta$ is defined by $\eta=\left\{\eta_{i}\right\}_{i=1}^{\infty}=\left\{\left(\int_{\Omega} f_{i} d \mu\right)^{q}\right\}_{i=1}^{\infty}$. S is given by functions like $\sum_{i=1}^{\infty} \lambda_{i} \chi_{A_{i}}$-where the sets $A_{i}$ are the support of the functions $f_{i}$ and the norms of these functions in $L^{p}(\nu)$ are given by

$$
\|f\|_{L^{p}(\nu)}:=\left(\sum_{i=1}^{\infty}\left|\lambda_{i}\right|^{q p} \eta_{i}\right)^{1 / q p}
$$

Therefore, $S$ is a subspace of $L^{1}(\nu)$ but not of $L^{p}(\nu)$ for any $p>1$.

The authors thank the anonymous referee for the careful reading of the paper and some comments and suggestions that have improved the paper.

\section{REFERENCES}

[1] O. Blasco, J. M Calabuig and E.A. Sánchez Pérez, p-variations of vector measures with respect to vector measures and integral representation of operators, Banach J. Math. Anal. 9,1 (2015), 273-285.

[2] J.M Calabuig, J. Rodríguez and E.A. Sánchez Pérez, Strongly embedded subspaces of p-convex Banach function spaces, Positivity 17(3) (2013), 775-791.

[3] N.L. Carothers and S.J. Dilworth, Subspaces of $L_{p, q}$, Proc. Am. Math. Soc. 104, 2, (1988), 537-545.

[4] J. Diestel and J. J. Uhl, Vector Measures, Math. Surveys, vol. 15, Amer. Math. Soc., Providence, RI, 1977.

[5] A. Fernández, F. Mayoral, F. Naranjo, C. Sáez and E.A. Sánchez-Pérez, Spaces of p-integrable functions with respect to a vector measure, Positivity 10 (2006), 1-16.

[6] T. Figiel, W. B. Johnson and L. Tzafriri, On Banach lattices and spaces having local unconditional structure with applications to Lorentz funtion spaces, J. Approx. Theory, 13 (1975), 395-412.

[7] J. Flores, F. L. Hernández, N. J. Kalton and P. Tradacete, Characterizations of strictly singular operators on Banach lattices, J. London Math. Soc.,(2) 79 (2009), 612-630.

[8] J. Flores, F. L. Hernández, E. M. Semenov and P. Tradacete, Strictly singular and power-compact operators on Banach lattices, Israel J. Math. 188,1 (2012), 323-352,

[9] I. Kadec, A. Pełczynski. Bases, Lacunary sequences and complemented subspaces in the spaces $L_{p}$, Studia Math. 21 (1962), 161-176. 
[10] N.J. Kalton, Banach spaces embedded into $L_{0}$, Israel J. Math. 52, (1985), 305-319.

[11] J. Lindenstrauss and L. Tzafriri, Classical Banach Spaces, II, Springer, Berlin, 1996.

[12] S.Ya. Novikov, Singularities of embedding operators between symmetric function spaces on [0,1], Math. Notes, 62 (4) (1997) 457-468.

[13] S. Okada, W.J. Ricker and E.A. Sánchez Pérez, Optimal domain and integral extension of operators acting in function spaces. Operator Theory: Advances and Applications, 180. Birkhäuser Verlag, Basel, 2008.

[14] S. Okada, W.J. Ricker, E.A. Sánchez Pérez, Lattice copies of $c_{0}$ and $\ell_{1}$ in spaces of integrable functions for a vector measure, Dissertationes Mathematicae, 500, (2014), 66.

[15] V.A. Rodin and E.M.Semyonov, Rademacher series in symmetric spaces, Anal. Math. 1 (2), (1975), 161-176.

[16] E. A. Sánchez Pérez, Compactness arguments for spaces of p-integrable functions with respect to a vector measure and factorization of operators through Lebesgue-Bochner spaces, Illinois J. Math. 453 (2001), 907-923.

[17] P. Tradacete, Subspace structure of Lorentz $L_{p, q}$ spaces and strictly singular operators, J. Math. Anal. Appl. 3671 (2010), 98-107.

[Pilar Rueda] Departamento de Análisis Matemático, Universidad de Valencia, 46100 Burjassot - Valencia, Spain, e-mail: pilar.rueda@uv.es

[Enrique A. Sánchez Pérez] Instituto Universitario de Matemática Pura y Aplicada, Universitat Politècnica de València, Camino de Vera s/n, 46022

Valencia, Spain, e-mail: easancpe@mat.upv.es 